\title{
From strong-field physics in and at nanoscale matter to photonics-based laser accelerators
}

Martin Kozák ${ }^{1,2}$, Takuya Higuchi ${ }^{1}$, Joshua McNeur ${ }^{1}$, Roy Shiloh ${ }^{1}$, Christian Heide ${ }^{1}$, Timo Paschen ${ }^{1}$, Peyman Yousefi ${ }^{1}$, Constanze Sturm ${ }^{1}$, Ang Li ${ }^{1}$, Johannes Illmer $^{1}$, Stefan Meier ${ }^{1}$, Norbert Schönenberger ${ }^{1}$, Philip Dienstbier ${ }^{1}$, Alexander Tafel ${ }^{1}$, Philipp Weber ${ }^{1}$, Robert Zimmermann ${ }^{1}$, Michael Seidling ${ }^{1}$, Anna Mittelbach $^{1}$, Jonas Heimerl', Timo Eckstein ${ }^{1}$, Martin Hundhausen ${ }^{1}$, Jürgen Ristein ${ }^{1}$ and Peter Hommelhoff ${ }^{1,3, *}$

${ }^{1}$ Department of Physics, Friedrich-Alexander-Universität Erlangen-Nürnberg (FAU), Staudtstraße 1, D-91058 Erlangen, Germany, EU

${ }^{2}$ now with Faculty of Mathematics and Physics, Charles University, Ke Karlovu 3, 12116 Prague 2, Czech Republic, EU

${ }^{3}$ associated with Max Planck Institute for the Science of Light (MPL), Staudtstraße 2, D-91058 Erlangen, Germany, EU

\begin{abstract}
New ways of controlling quasi-free and free electrons by means of phase-controlled ultrashort laser pulses are demonstrated: from strongfield physics in the conducting 2-d material graphene and at the surface of nanostructures, to laser acceleration of free electrons with a nanophotonic structure, and the demonstration of the longitudinal Kapitza-Dirac effect.
\end{abstract}

Twenty-five years after the broad acceptance of the recollision picture in atomic strongfield physics [1,2], introducing optical-field-driven control over electrons, a similar level of control has not long ago been introduced to solid matter. Until recently, research on electron dynamics inside of solids has been limited to dielectrics and semiconductors [3]. We have recently shown the transition from the weak-field to the strong-field regime in the conductor graphene, a 2-dimensional layer of carbon atoms. In the strong-field regime, we observe coherent electron dynamics and quantum path interference in the form of repeated Landau-Zener transitions, known as Landau-Zener-Stückelberg interferometry [4].

Strong-field physics at the surface of nanoscale structures has been demonstrated several years ago [5]. Quantum-path interference with a very high degree of coherence is demonstrated in photoemission from metal tips [6], as well as the tell-tale plateau structure, indicating electronic recollision, from nanoscale structures with other dimensionality.

In the second part of the talk we have discussed controlling electrons in free space with the help of two-colour laser fields and with the help of dielectric, nano-photonic structures. Two-colour laser fields impinging on an electron beam in a scanning electron microscope under two different angles allow us to generate an optical traveling wave co-propagating with the femtosecond electron bunch. This way, an efficient momentum transfer from the light field to the electrons can take place. We demonstrate an acceleration gradient of 2.2 $\mathrm{GeV} / \mathrm{m}$, which is one to two orders of magnitude larger than in modern particle accelerators [7]. This represents also a demonstration of the longitudinal version of the Kapitza-Dirac effect. We furthermore show that a pulse train with individual pulse durations shorter than 300 attoseconds results [8].

\footnotetext{
* Corresponding author: peter.hommelhoff@fau.de
} 
With laser fields properly shaped by transparent nano-photonic structures, electrons in the optical near-field of these structures can be accelerated efficiently, and in great similarity to today's particle accelerators operating principle, namely with phasesynchronous acceleration, but here at optical frequencies. The proof-of-concept experiments have been performed several years ago [9]. We showed that we can not only accelerate with gradients approaching $1 \mathrm{GeV} / \mathrm{m}$ but also deflect, focus and streak electrons [10]. Thus, we have full control over a free-space electron beam with optical forces only and may now build a miniaturized particle accelerator composed of nano-photonic building blocks. This is what the Accelerator on a Chip International Program (ACHIP) is pursuing [11].

We acknowledge funding from the Gordon and Betty Moore Foundation (Accelerator on a Chip International Program), the European Research Council (Consolidator Grant Near Field Atto), the Deutsche Forschungsgemeinschaft (Collaborative Research Centre SFB953 Carbon Allotropes and Priority Program SPP1840 QUTIF), the Bundesministerium für Bildung und Forschung (05K16WEC),

\section{References}

1. K. J. Schafer, B. Yang, L. F. DiMauro, and K. C. Kulander, Phys. Rev. Lett. 70, 1599 (1993), P. B. Corkum, Phys. Rev. Lett. 71, 1994 (1993)

2. K. Schafer, M. Vrakking, Zhiyi Wei, eds., Special Issue "25 years of recollision physics", J. Phys. B: At. Mol. Opt. Phys. 50, 170201 (2017); P. Hommelhoff, M. Vrakking, Symposium "25 years of recollision physics", DPG spring meeting, March 4 $-9,2018$, in Erlangen

3. S. Ghimire, DiChiara, A. D., Sistrunk, E., Agostini, P., DiMauro, Louis F., Reis, D. A. Nat. Phys. 7, 138-141 (2011); T. Paasch-Colberget al., Nature 493, 70-74 (2012); M. Schultze et al., Science 346, 1348-1352 (2014); Vampa, G., Hammond, T. J., Thiré, N., Schmidt, B. E., Légaré, F., McDonald, C. R., Brabec, T., Corkum, P. B. Nature 522, 462-464 (2015); Langer, F., et al., Nature 533, 225-229 (2016); M. Lucchine et al., Science 353, 916 (2016)

4. T. Higuchi, Heide, C., Ullmann, K., Weber, H.B., Hommelhoff, P., Light-field-driven currents in graphene, Nature 550, 224 (2017).

5. R. Bormann, M. Gulde, A. Weismann, S. V. Yalunin, C. Ropers, Phys. Rev. Lett. 105, 147601 (2010); M. Schenk, M. Krüger, P. Hommelhoff, Phys. Rev. Lett. 105, 257601 (2010); M. Krüger, M. Schenk, P. Hommelhoff, Nature 475, 78 (2011); G. Herink, D. R. Solli, M. Gulde, C. Ropers, Nature 483, 190 (2012); B. Piglosiewizc et al., Nat. Phot. 8, 37 (2014); P. Hommelhoff, M. Kling, (eds.), Attosecond Nanophysics, Wiley 2015; M. Ciappina et al., Rep. Progr. Phys. 80, 054401 (2017)

6. M. Förster, T. Paschen, M. Krüger, Chr. Lemell, G. Wachter, F. Libisch, Th. Madlener, J. Burgdörfer, P. Hommelhoff, Phys. Rev. Lett. 117, 217601 (2016); T. Paschen, M. Förster, M. Krüger, Chr. Lemell, G. Wachter, F. Libisch, Th. Madlener, J. Burgdörfer, P. Hommelhoff, J. Mod. Opt. 64, 1054 (2017)

7. M. Kozák, T. Eckstein, N. Schönenberger, P. Hommelhoff, Nature Physics 14, 121 (2018)

8. M. Kozák, N. Schönenberger, P. Hommelhoff, Phys. Rev. Lett. 120, 103203 (2018)

9. E. Peralta et al., Nature 503, 91 (2013); J. Breuer, P. Hommelhoff, Phys. Rev. Lett. 111, 134803 (2013) 
10. M. Kozák et al., Optics Letters 41, 3435 (2016); M. Kozák et al., Nature Communications 8, 14342 (2017); M. Kozák et al., Optics Express 25, 19195 (2017); J. McNeur et al., Optica 5, 687 (2018); P. Yousefi et al., to be published

11. http://achip.stanford.edu 